\title{
Efeitos da dopagem com gadolínia na densificação e nas propriedades elétricas de soluções sólidas $\mathrm{Ce}_{0,99-\mathrm{x}} \mathrm{Gd}_{\mathrm{x}} \mathrm{Cu}_{0,01} \mathrm{O}_{2-\delta}$
}

\section{(Effects of gadolinia doping on densification and electrical properties of $C e_{0.99-x} G d_{x} C u_{0.01} O_{2-\delta}$ solid solutions)}

\author{
T. H. S. Silva', C. G. M. Lima ${ }^{1}$, R. P. S. Dutra', F. de M. Aquino', J. P. F. Grilo ${ }^{3}$, S. Rajesh ${ }^{3}$, D. A. Macedo ${ }^{1}$ \\ ${ }^{1}$ Programa de Pós-Graduação em Ciência e Engenharia de Materiais; ${ }^{2}$ Programa de Pós-Graduação em \\ Energias Renováveis, UFPB, João Pessoa, PB, Brasil 58051-900 \\ ${ }^{3}$ Departamento de Engenharia de Materiais e Cerâmica, Universidade de Aveiro, 3810-193 Aveiro, Portugal \\ thamyscira@gmail.com,chrystian.m.lima@hotmail.com, ricardopsd@gmail.com,flavia@cear.ufpb.br,grilo. \\ jpf@gmail.com,rajeshayr@gmail.com,damaced@gmail.com
}

\begin{abstract}
Resumo
Soluções sólidas à base de céria $\left(\mathrm{CeO}_{2}\right)$ são materiais promissores para aplicação como eletrólitos de células a combustível de óxido sólido. Apesar das boas propriedades elétricas, um dos principais inconvenientes destes materiais são as altas temperaturas de sinterização (normalmente acima de $1400{ }^{\circ} \mathrm{C}$ ) necessárias para completa densificação. Com o intuito de melhorar a sinterabilidade da céria, óxidos de metais de transição com baixo ponto de fusão têm sido usados como aditivos de sinterização. Neste contexto, o objetivo deste trabalho foi avaliar o efeito do teor de gadolínia $\left(\mathrm{Gd}_{2} \mathrm{O}_{3}\right)$ na densificação e propriedades elétricas de soluções sólidas de céria com $\mathrm{CuO}$ como aditivo de sinterização. Pós com composições nominais $\mathrm{Ce}_{0,99-\mathrm{x}} \mathrm{Gd}_{\mathrm{x}} \mathrm{Cu}_{0,01} \mathrm{O}_{2-\delta}(\mathrm{x}=0,0,2$ e 0,3$)$ foram sintetizados pelo método dos precursores poliméricos, calcinados a $500{ }^{\circ} \mathrm{C}$ e caracterizados por difratometria de raios $\mathrm{X}$ (DRX). A densidade relativa foi avaliada em amostras sinterizadas entre 950 e $1050{ }^{\circ} \mathrm{C}$. As propriedades elétricas foram estudadas por espectroscopia de impedância. Os resultados de DRX indicaram a obtenção de soluções sólidas com estrutura cúbica do tipo fluorita. O uso de materiais nanoparticulados associado a um aditivo de sinterização permitiu a obtenção de cerâmicas com densidades relativas superiores a $92 \%$ após sinterização em temperaturas tão baixas quanto $1000{ }^{\circ} \mathrm{C}$. A condutividade elétrica total foi otimizada em amostras com $20 \%$ em mol de $\mathrm{Gd}_{2} \mathrm{O}_{3}$.

Palavras-chave: céria dopada com gadolínia, precursores poliméricos, aditivo de sinterização, condutividade elétrica.
\end{abstract}

Abstract

Ceria $\left(\mathrm{CeO}_{2}\right)$ based solid solutions are promising materials for use as solid oxide fuel cells electrolytes. Despite good electrical properties, one of the major drawbacks of these materials are the high sintering temperatures (typically above $1400{ }^{\circ} \mathrm{C}$ ) required for full densification. In order to improve the ceria sinterability, transition metal oxides with low melting point have been used as sintering aid. In this context, the aim of this study was to evaluate the effect of gadolinia $\left(\mathrm{Gd}_{2} \mathrm{O}_{3}\right)$ content on densification and electrical properties of ceria solid solutions with $\mathrm{CuO}$ as a sintering aid. Powders with nominal compositions $\mathrm{Ce} e_{0.99-x} \mathrm{Gd} \mathrm{Cu}_{x} \mathrm{O}_{0.01} \mathrm{O}_{2-\delta}$ $(x=0,0.2$ and 0.3$)$ were synthesized by the polymeric precursor method, calcined at $500{ }^{\circ} \mathrm{C}$ and characterized by $X$-ray diffraction (XRD). The relative density was evaluated in samples sintered between 950 and $1050{ }^{\circ} \mathrm{C}$. The electrical properties were evaluated by impedance spectroscopy. The XRD results indicated the attainment of solid solutions with cubic fluorite type structure. The use of nanoparticle materials associated with a sintering aid allowed to obtain ceramics with relative densities higher than $92 \%$ after sintering at temperatures as low as $1000{ }^{\circ} \mathrm{C}$. The total electrical conductivity was enhanced in samples with $20 \mathrm{~mol}_{\mathrm{O}} \mathrm{Gd} \mathrm{O}_{3}$.

Keywords: gadolinia doped ceria, polymeric precursor, sintering aid, electrical conductivity.

\section{INTRODUÇÃO}

As células a combustível de óxido sólido (CCOS), dispositivos eletroquímicos que convertem a energia química da reação de combustíveis em eletricidade, vêm se destacando como uma das principais promessas tecnológicas de produção de energia limpa, por apresentar flexibilidade no uso de combustíveis e característica modular [1-4]. A unidade básica de uma CCOS consiste essencialmente de dois eletrodos porosos (catodo e anodo) separados por um eletrólito denso. No catodo ocorre a reação de redução do gás oxidante, com formação de íons de $\mathrm{O}^{2-}$ que migram através do eletrólito denso até chegar à estrutura porosa do anodo. Chegando ao anodo, os íons de $\mathrm{O}^{2-}$ promovem a reação de oxidação do combustível. Os produtos dessa reação são corrente elétrica e água, quando hidrogênio é usado como combustível. Células convencionais com eletrólito de zircônia estabilizada com ítria (ZEI) necessitam operar a 
altas temperaturas $\left(\sim 1000{ }^{\circ} \mathrm{C}\right)$ a fim de manter a resistência específica de área destes eletrólitos em níveis aceitáveis. Como as altas temperaturas de operação estão associadas aos problemas relacionados à degradação de materiais e aos elevados custos de fabricação, nos últimos anos grande parte dos esforços de pesquisa tem se concentrado em reduzir a temperatura de operação das células para a faixa entre 600 e $800{ }^{\circ} \mathrm{C}$, ou até mesmo para temperaturas próximas de 500 ${ }^{\circ} \mathrm{C}[5,6]$. Embora a redução da temperatura de operação minimize os custos com materiais e processos de fabricação, ela também favorece o aumento na resistência do eletrólito e diminui as taxas das reações eletrocatalíticas dos eletrodos. Estes fatores resultam em redução da tensão e potência de saída da célula.

Para solucionar este problema, materiais cerâmicos à base de céria $\left(\mathrm{CeO}_{2}\right)$ dopada com terras raras $\left(\mathrm{Y}^{3+}, \mathrm{Gd}^{3+}\right.$, $\mathrm{Sm}^{3+}$, etc.) têm sido propostos como eletrólitos de células a combustível de óxido sólido de temperatura intermediária (operando entre 600 e $800{ }^{\circ} \mathrm{C}$ ). Especialmente a céria dopada com gadolínia $\left(\mathrm{Ce}_{1-x} \mathrm{Gd}_{\mathrm{x}} \mathrm{O}_{2-x / 2}-\mathrm{CGO}\right)$ apresenta condutividade iônica maior que a da ZEI na faixa de temperatura de 300 a $700{ }^{\circ} \mathrm{C}$, sendo considerada uma forte candidata para aplicação como eletrólito em temperaturas intermediárias [7]. O dopante usado na céria deve ser escolhido de tal forma que o seu raio iônico (valor ideal próximo a $1,04 \AA$ A) cause pouca deformação da rede da céria, maximizando a condutividade elétrica do eletrólito. Os dopantes que proporcionam maiores condutividades iônicas são os íons de samário $\left(\mathrm{Sm}^{3+}\right.$, raio iônico de 1,079 Å) e gadolínio $\left(\mathrm{Gd}^{3+}\right.$, raio iônico de 1,053 $\AA$ ), pois seus raios iônicos não provocam nem expansão e nem contração dos parâmetros de rede da céria [8-10]. O aumento do teor de dopantes não significa necessariamente aumento proporcional na condutividade, mesmo o teor de dopantes estando abaixo do limite de solubilidade. Uma possível diminuição de condutividade com a adição de dopantes pode ser explicada pelo aumento da interação entre vacâncias de oxigênio e cátions substituintes, com cargas opostas. Esta interação limita a mobilidade das vacâncias, reduzindo a condutividade iônica. A literatura aponta que a otimização da condutividade iônica de eletrólitos à base de céria ocorre para teores de dopante em torno de $20 \%$ em mol [7, 11, 12].

A baixa sinterabilidade de cerâmicas à base de céria requer altas temperaturas de processamento (1400 a 1600 $\left.{ }^{\circ} \mathrm{C}\right)$ a fim de atingir a densificação aceitável $(>92 \%)$ para uso como eletrólito sólido em CCOS. Entretanto, nestas condições de tratamento térmico a céria altera seu estado de valência de +4 para +3 . Este fenômeno de redução da céria afeta a sua condutividade (torna-se condutora eletrônica) e resistência mecânica (surgimento de microtrincas) [13]. No intuito de solucionar este problema, o uso de materiais nanoparticulados e a adição de pequenos teores de aditivos de sinterização têm sido alternativas largamente utilizadas [14-17]. Os materiais nanoparticulados ainda necessitam ser processados em torno de $1200{ }^{\circ} \mathrm{C}$ para atingir total densificação [18]. O aditivo de sinterização deve ser capaz de melhorar a sinterabilidade através da formação de uma fase líquida. Basicamente, o mecanismo consiste na fase líquida molhar os grãos sólidos por meio de uma força capilar que aproxima as partículas, acelerando a densificação $[13,19]$. Neste contexto, este trabalho teve como objetivo principal avaliar o efeito do teor de gadolínia $\left(\mathrm{Gd}_{2} \mathrm{O}_{3}\right)$ na estrutura cristalina, microestrutura e propriedades elétricas de eletrólitos sólidos à base de céria $\left(\mathrm{CeO}_{2}\right)$ codopados com óxido de cobre II (CuO), usado como aditivo de sinterização. Os materiais particulados, sintetizados pelo método dos precursores poliméricos, foram investigados utilizando técnicas avançadas de caracterização de materiais. O uso combinado de nanopartículas e aditivo de sinterização $(\mathrm{CuO})$ permitiu a obtenção de soluções sólidas cerâmicas com microestruturas densas em temperaturas de processamento tão baixas quanto $1000^{\circ} \mathrm{C}$.

\section{EXPERIMENTAL}

Pós de céria dopada com diferentes teores de gadolínia, de acordo com a composição $\mathrm{Ce}_{0,99-\mathrm{x}} \mathrm{Gd}_{\mathrm{x}} \mathrm{Cu}_{0,01} \mathrm{O}_{2-\delta}(\mathrm{x}=0,0,2$ e 0,3$)$, e codopada com $1 \%$ em mol de $\mathrm{CuO}$, usado como aditivo de sinterização, foram preparados pelo método dos precursores poliméricos seguindo a metodologia usada em trabalho anterior [20] As resinas poliméricas foram submetidas a um tratamento térmico a $300{ }^{\circ} \mathrm{C}$ por 2 h usando taxa de aquecimento de $1{ }^{\circ} \mathrm{C} / \mathrm{min}$. Os materiais resultantes foram desagregados em almofariz de ágata para a formação dos pós precursores. Por fim, os pós precursores foram submetidos a uma etapa de calcinação a $500{ }^{\circ} \mathrm{C}$ por $1 \mathrm{~h}$ usando taxa de aquecimento de $3{ }^{\circ} \mathrm{C} / \mathrm{min}$. A estrutura cristalina dos pós calcinados foi analisada pela técnica de difratometria de raios X (DRX), para determinar o parâmetro de rede, tamanho de cristalito e densidade teórica. Os parâmetros de ensaio foram varredura angular $2 \theta$ entre 20 e $80^{\circ}$, passo de $0,02^{\circ}$ e tempo de contagem de $2 \mathrm{~s}$ por passo usando radiação monocromática $\mathrm{K} \alpha$ do cobre $(\lambda=1,5418 \AA$ A $)$. O refinamento Rietveld dos dados de difração foi realizado utilizando o software MAUD, sendo que para cada composição utilizou-se um padrão ICSD específico. As densidades teóricas para cada composição preparada foram calculadas usando a equação:

$\rho_{\mathrm{t}}=\left(\frac{4}{\mathrm{~N}_{\mathrm{A}} \cdot \mathrm{a}^{3}}\right)\left[(1-(\mathrm{x}-\mathrm{y})) \mathrm{M}_{\mathrm{Ce}}+\mathrm{xM}_{\mathrm{Gd}}+\mathrm{yM}_{\mathrm{Cu}}+\left(2-\frac{\mathrm{x}}{2}-\frac{\mathrm{y}}{2}\right) \mathrm{M}_{\mathrm{O}}\right]$

onde, $N_{A}$ é o número de Avogadro, $a$ é o parâmetro de rede, $M$ é a massa atômica de cada elemento, $x$ é o teor de gadolínio e $y$ é o teor de cobre.

Pastilhas cerâmicas foram preparadas para caracterização de densidade relativa e espectroscopia de impedância. A confecção das partilhas (diâmetro de $10 \mathrm{~mm}$ ) foi realizada por prensagem uniaxial em prensa hidráulica utilizando-se pressão de $127 \mathrm{MPa}$, mantida por $30 \mathrm{~s}$. Após a prensagem, as pastilhas foram sinterizadas em forno tipo mufla com atmosfera de ar a 950,1000 e $1050{ }^{\circ} \mathrm{C}$, usando taxa de aquecimento de $3{ }^{\circ} \mathrm{C} / \mathrm{min}$ e patamar de $5 \mathrm{~h}$. O princípio de Arquimedes (imersão em água por $24 \mathrm{~h}$ ) foi utilizado para obter os valores de massa específica aparente (MEA). Os 
cálculos de MEA e densidade relativa $\left(\rho_{\mathrm{r}}\right)$ foram realizados usando as equações:

$$
\begin{aligned}
& M E A=\frac{P_{S}}{P_{U}-P_{I}} \times \rho_{\text {água }} \\
& \rho_{r}=\frac{\text { MEA }}{\rho_{t}}
\end{aligned}
$$

onde, $\mathrm{P}_{\mathrm{s}}$ é a massa seca, $\mathrm{P}_{\mathrm{u}}$ é a massa úmida, $\mathrm{P}_{\mathrm{i}}$ é a massa imersa, $\rho_{\text {água }}$ é a densidade da água (considerada $1 \mathrm{~g} / \mathrm{cm}^{3}$ ) e $\rho_{\mathrm{t}}$ é a densidade teórica obtida por DRX. As superfícies de fratura foram analisadas usando um microscópio eletrônico de varredura (MEV, mod. 1430 da LEO). A caracterização elétrica de pastilhas sinterizadas a $1000{ }^{\circ} \mathrm{C}$ por $5 \mathrm{~h}$ foi realizada por espectroscopia de impedância entre 400 e 850 ${ }^{\circ} \mathrm{C}$ em ar, usando um analisador de impedância HP 4284A, um porta-amostra e um forno tubular. A faixa de frequência utilizada foi de $20 \mathrm{~Hz}$ a $1 \mathrm{MHz}$, com amplitude de sinal de 0,5 V. Antes das medidas elétricas, eletrodos porosos de $\mathrm{Pt}$ foram preparados usando pasta de Pt. A pasta foi depositada em faces paralelas das pastilhas e curada a $1000{ }^{\circ} \mathrm{C}$ por $1 \mathrm{~h}$ em ar. Os resultados de impedância foram analisados utilizando o programa ZView (Scribner Associates).

\section{RESULTADOS E DISCUSSÃO}

A técnica de difratometria de raios X (DRX) foi utilizada para investigar as características estruturais (estrutura cristalina, parâmetro de rede e tamanho de cristalito) e a

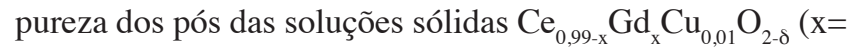
$0,0,2$ e 0,3 ) obtidos pelo método dos precursores poliméricos e calcinados a $500{ }^{\circ} \mathrm{C}$ por $1 \mathrm{~h}$. Os difratogramas de raios $\mathrm{X}$ refinados apresentados na Fig. 1 evidenciaram que as composições com $1 \%$ em mol de $\mathrm{CuO}$ e diferentes teores do íon $\mathrm{Gd}^{3+}$ apresentam picos característicos da estrutura cúbica tipo fluorita (grupo espacial $F m 3 m$ ), a mesma da céria (óxido de cério). A Fig. 1 também ilustra a comparação entre os difratogramas calculado (por Rietveld), obtido experimentalmente e residual (diferença entre calculado e experimental) para as três composições em estudo. Os picos de difração foram indexados com base nas cartas JPCDS 43-1002 $\left(\mathrm{CeO}_{2}\right), 75-0162\left(\mathrm{Ce}_{0,8} \mathrm{Gd}_{0,2} \mathrm{O}_{1,90}, \mathrm{CGO} 20\right)$ e 75$0163\left(\mathrm{Ce}_{0,7} \mathrm{Gd}_{0,3} \mathrm{O}_{1,85}, \mathrm{CGO} 30\right)$, para os pós com 0, 20 e $30 \%$ em mol de gadolínia, respectivamente. A ausência de fases secundárias, dentro do limite de detecção da difratometria de raios $\mathrm{X}$, atesta a pureza das soluções sólidas sintetizadas, sugerindo que os teores adotados dos íons $\mathrm{Cu}^{2+}$ e $\mathrm{Gd}^{3+}$ não ultrapassaram os seus limites de solubilidade na rede da céria. $\mathrm{O}$ efeito do teor de gadolínio sobre o alargamento e consequente diminuição da intensidade dos picos de difração é notável, sendo uma indicação da redução do tamanho de cristalito. Com base nesta observação, esperase que o pó com a maior dopagem de gadolínia apresente o menor tamanho de cristalito, o que será posteriormente confirmado por refinamento Rietveld. Uma vez que os

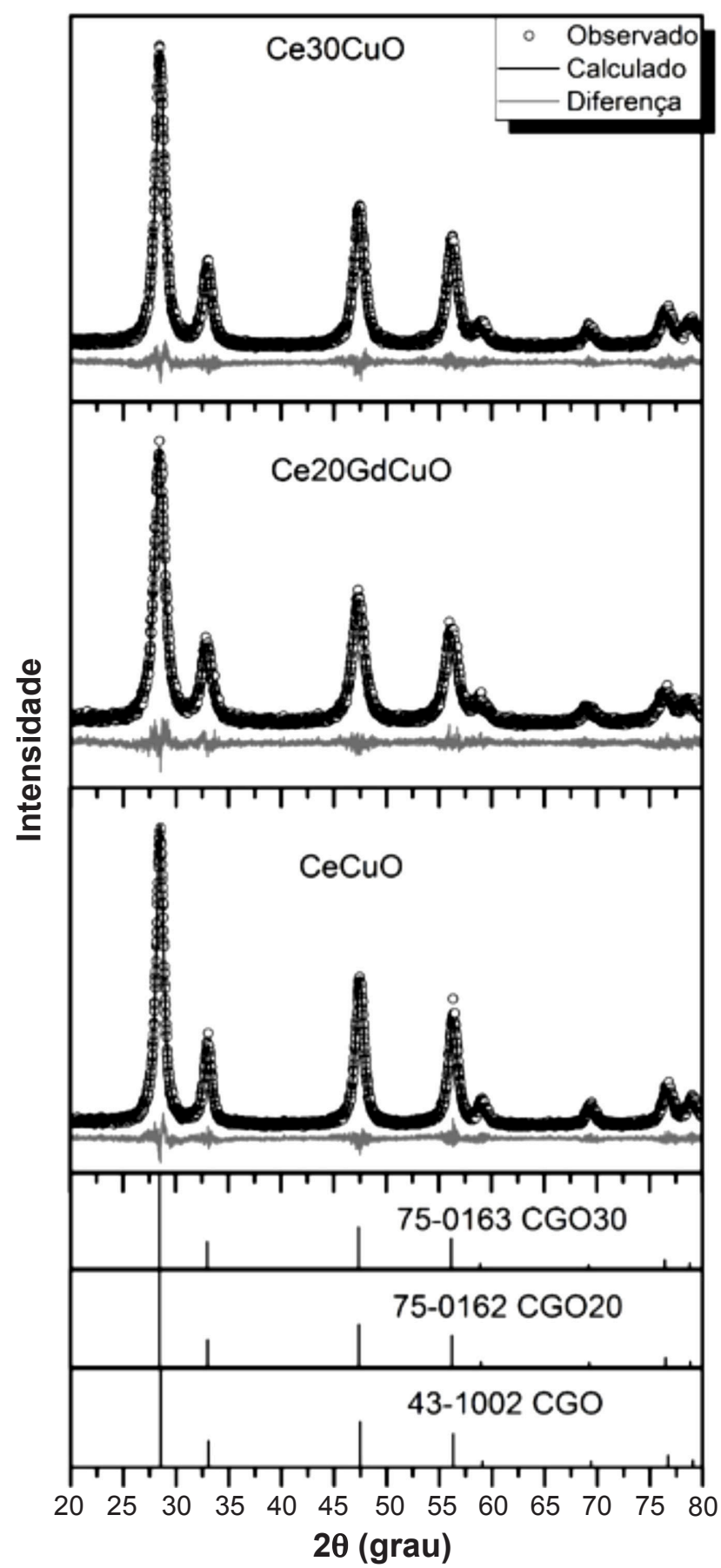

Figura 1: Difratogramas de raios $\mathrm{X}$ refinados dos pós calcinados a $500{ }^{\circ} \mathrm{C}$ por $1 \mathrm{~h}$.

[Figure 1: Refined X-ray diffraction patterns of powders calcined at $500{ }^{\circ} \mathrm{C}$ for $\left.1 \mathrm{~h}.\right]$

íons $\mathrm{Ce}^{4+}$ são inicialmente substituídos por $\mathrm{Cu}^{2+}$, formando $\mathrm{Ce}_{0,99} \mathrm{Cu}_{0,01} \mathrm{O}_{2-\delta}$, e posteriormente substituídos por teores crescentes de $\mathrm{Gd}^{3+}$, originando soluções sólidas $\mathrm{Ce}-\mathrm{Gd}$ $\mathrm{Cu}-\mathrm{O}$, há um progressivo aumento das vacâncias de oxigênio (indicadas pelo símbolo $\delta$ ).

O refinamento Rietveld dos dados de difração, realizado utilizando o programa computacional Maud e cartas JCPDS específicas para cada composição (mencionadas acima), permitiu a obtenção dos parâmetros cristalográficos 
Tabela I - Parâmetros cristalográficos e índices de refinamento para as composições calcinadas a $500^{\circ} \mathrm{C}\left(\mathrm{Ce}_{0.99-\mathrm{x}} \mathrm{Gd}_{\mathrm{x}} \mathrm{Cu}_{0.01} \mathrm{O}_{2-\delta}\right)$. [Table I - Crystallographic parameters and refinement indexes for compositions calcined at $500{ }^{\circ} \mathrm{C}\left(\mathrm{Ce}_{0.99-x} \mathrm{Gd}_{x} \mathrm{Cu} u_{0.01} \mathrm{O}_{2-\delta}\right)$.]

\begin{tabular}{cccc}
\hline Parâmetro & $\mathrm{x}=0$ & $\mathrm{x}=0,20$ & $\mathrm{x}=0,30$ \\
\hline $\mathrm{a}=\mathrm{b}=\mathrm{c}(\mathrm{nm})$ & $0,5410(3)$ & $0,5425(2)$ & $0,5432(8)$ \\
Volume $\left(\AA^{3}\right)$ & 158,36 & 159,67 & 160,35 \\
$\mathrm{D}_{\text {DRX }}(\mathrm{nm})$ & 14,89 & 10,54 & 7,63 \\
$\mathrm{R}_{\text {wp }}(\%)$ & 13,31 & 12,40 & 12,26 \\
$\mathrm{R}_{\text {exp }}(\%)$ & 11,02 & 10,92 & 10,92 \\
$\chi^{2}$ & 1,21 & 1,13 & 1,12 \\
\hline
\end{tabular}

Tabela II - Densidade teórica $\left(\mathrm{g} / \mathrm{cm}^{3}\right)$ para as composições estudadas $\left(\mathrm{Ce}_{0,99-\mathrm{x}} \mathrm{Gd}_{\mathrm{x}} \mathrm{Cu}_{0,01} \mathrm{O}_{2-\delta}\right)$.

[Table II - Theoretical density $\left(\mathrm{g} / \mathrm{cm}^{3}\right)$ for the studied compositions $\left(\mathrm{Ce}_{0.99-x} \mathrm{Gd}_{x} \mathrm{Cu}_{0.01} \mathrm{O}_{2-\delta}\right)$.]

\begin{tabular}{ccc}
\hline $\mathrm{x}=0$ & $\mathrm{x}=0,20$ & $\mathrm{x}=0,30$ \\
\hline 7,21 & 7,32 & 7,33 \\
\hline
\end{tabular}

[parâmetro de rede $(n m)$, volume da célula unitária $\left(\AA^{3}\right)$ e tamanho de cristalito - $\mathrm{D}_{\mathrm{DRX}}(\mathrm{nm})$, e índices de refinamento - $\mathrm{R}_{\mathrm{wp}}, \mathrm{R}_{\mathrm{exp}}$ e $\chi^{2}$ ] apresentados na Tabela I. A qualidade dos refinamentos pôde ser avaliada pelos valores do índice $\chi^{2}$, que expressa o quociente $\mathrm{R}_{\text {wp }} / \mathrm{R}_{\text {exp }}$. Quanto mais o valor de $\chi^{2}$ se aproxima da unidade, melhor é a qualidade do refinamento. Portanto, os baixos valores de $\chi^{2}$ (variando entre 1,12 e 1,21 , Tabela I) encontrados para as diversas composições em estudo foram coerentes com os bons ajustes gráficos apresentados na Fig. 1. Os resultados do refinamento indicaram que o parâmetro de rede da céria dopada com $1 \%$ em mol de $\mathrm{CuO}\left(\mathrm{Ce}_{0,99} \mathrm{CuO}_{2-\delta}\right)$ foi próximo ao da céria pura (a $=0,5411 \mathrm{~nm}$, de acordo com a carta JCPDS 43-1002) e as soluções sólidas com sucessivas adições de gadolínia experimentaram um crescente aumento do parâmetro de rede e, consequentemente, do volume da célula unitária, como previamente mencionado com base no alargamento dos picos de difração. Essa expansão da estrutura foi devido à diferença entre os raios iônicos do $\mathrm{Gd}^{3+}(0,105$ $\mathrm{nm})$ e do $\mathrm{Ce}^{4+}(0,097 \mathrm{~nm})$ [21], ou seja, a incorporação do íon trivalente (com maior raio iônico) causou deformação uniforme na estrutura. Os tamanhos de cristalito $\left(D_{\mathrm{DRX}}=12\right.$ $15 \mathrm{~nm}$ ) foram menores que valores típicos reportados na literatura para pós obtidos pelo método da combustão (40-50 nm) [22] e por um método de complexação de citratos (53 nm) [23], ambos preparados sob condições de calcinação similares às usadas neste estudo. É importante mencionar que pequenos tamanhos de cristalito/partícula tendem a proporcionar maiores densidades nas cerâmicas, além de reduzir a temperatura necessária para máxima densificação. Com base nos resultados dos parâmetros de rede foi possível calcular as densidades teóricas de cada composição usando a Equação A. Os resultados são apresentados na Tabela II.
O aumento da densidade teórica com a incorporação de gadolínio, e em relação à amostra dopada apenas com íon $\mathrm{Cu}$, é outra forte evidência da formação de soluções sólidas.

Na Fig. 2 são apresentadas as superfícies de fratura de
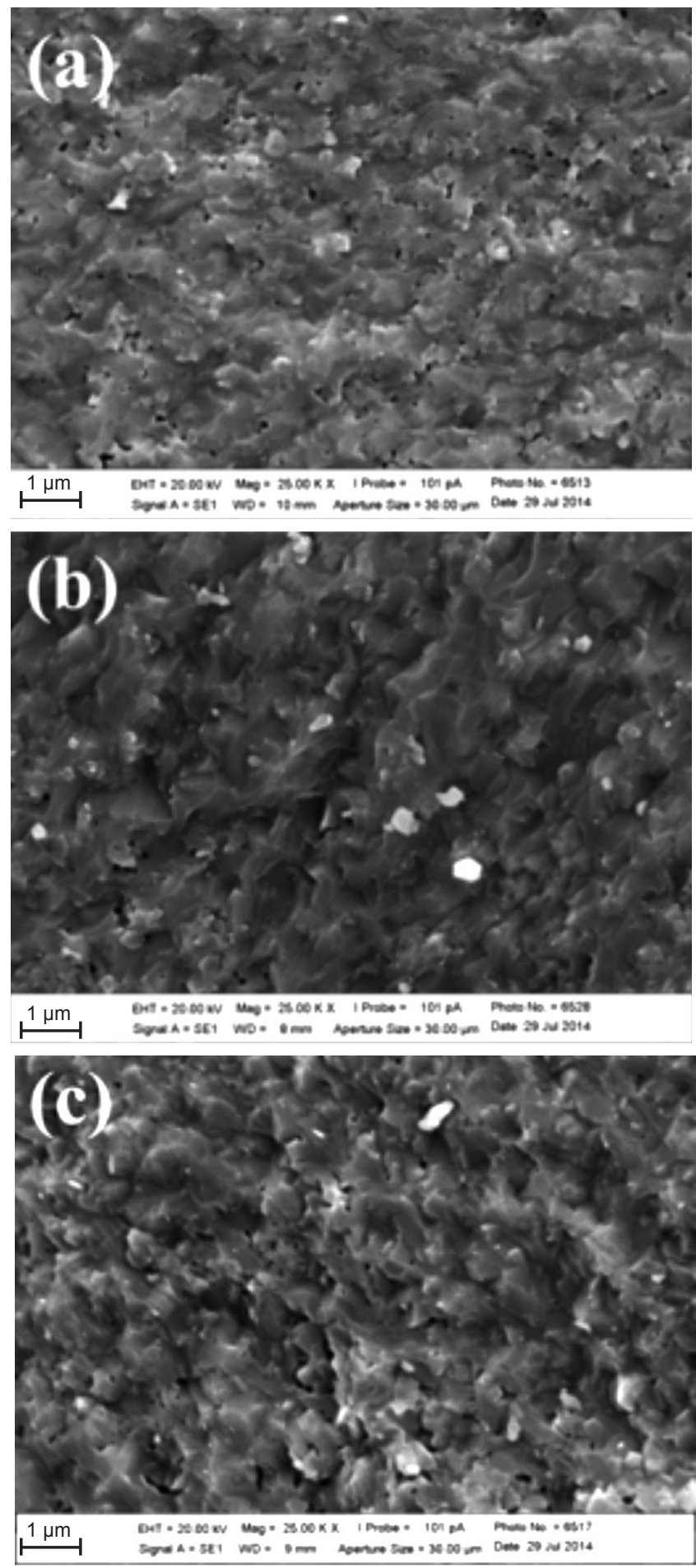

Figura 2: Micrografias obtidas por microscopia eletrônica de varredura das superfícies de fratura das amostras de composições $\mathrm{Ce}_{0,99-\mathrm{x}} \mathrm{Gd}_{\mathrm{x}} \mathrm{Cu}_{0,01} \mathrm{O}_{2-\delta}$ sinterizadas a $1000{ }^{\circ} \mathrm{C}$ : (a) $\mathrm{x}=0$, (b) $\mathrm{x}=0,2 \mathrm{e}$ (c) $\mathrm{x}=0,3$.

[Figure 2: SEM micrographs of fracture surfaces of samples with compositions $\mathrm{Ce}{ }_{0.99-x} \mathrm{Gd}_{x} \mathrm{Cu}_{0.01} \mathrm{O}_{2-\delta}$ sintered at $1000{ }^{\circ} \mathrm{C}:$ (a) $\mathrm{x}=0$, (b) $x=0.2$, and (c) $x=0.3$.] 
Tabela III - Densidade relativa (\%) em função da temperatura de sinterização e do teor de gadolínia $\left(\mathrm{Ce}_{0,99-\mathrm{x}} \mathrm{Gd}_{\mathrm{x}} \mathrm{Cu}_{0,01} \mathrm{O}_{2-\delta}\right)$. [Table III - Relative density (\%) as a function of sintering temperature and gadolinia content $\left(\mathrm{Ce}_{0.99-x} \mathrm{Gd}_{x} \mathrm{Cu}_{0.01} \mathrm{O}_{2-\delta}\right)$.]

\begin{tabular}{cccc}
\hline Temperatura $\left({ }^{\circ} \mathrm{C}\right)$ & $\mathrm{x}=0$ & $\mathrm{x}=0,20$ & $\mathrm{x}=0,30$ \\
\hline 950 & 69,01 & 90,57 & 91,50 \\
1000 & 92,01 & 92,54 & 95,00 \\
1050 & 94,10 & 95,41 & 95,10 \\
\hline
\end{tabular}

amostras de composições $\mathrm{Ce}_{0,99-\mathrm{x}} \mathrm{Gd}_{\mathrm{x}} \mathrm{Cu}_{0,01} \mathrm{O}_{2-\delta}(\mathrm{x}=0,0,2 \mathrm{e}$ $0,3)$ sinterizadas a $1000^{\circ} \mathrm{C}$ por $5 \mathrm{~h}$. As amostras apresentaram microestruturas características de alta densificação, ainda que pequenos poros fechados foram observados. A porosidade fechada residual foi maior na amostra livre de gadolínia. Pelo princípio de Arquimedes foi possível estudar os efeitos da temperatura de sinterização e do teor de gadolínia na densidade relativa. Os resultados são apresentados na Tabela III. Os resultados mostraram que o incremento na temperatura de sinterização provocou maior densificação das cerâmicas, como esperado. A ausência do íon gadolínio associada à menor temperatura de sinterização $\left(950^{\circ} \mathrm{C}\right)$ permitiu a obtenção de densidade relativa tão baixa quanto $69 \%$, valor muito inferior ao mínimo esperado para aplicação como eletrólito solido em célula a combustível ( $92 \%)$. Para este tipo de aplicação, a densidade relativa destes materiais deve ser a maior possível, tendo em vista o objetivo primordial de separar os gases combustível e oxidante presentes nos compartimentos de anodo e catodo, respectivamente. Estes resultados estão em bom acordo com a análise microestrutural qualitativa realizada por MEV. De um modo geral, a densidade relativa tendeu a aumentar com o aumento do teor de gadolínia, variando entre 92\% (livre de $\mathrm{Gd}^{3+}$ ) e $95 \%$ (maior teor de $\mathrm{Gd}^{3+}$ ) a $1000{ }^{\circ} \mathrm{C}$.

A caracterização elétrica de amostras selecionadas foi realizada por espectroscopia de impedância, em atmosfera de ar entre 400 e $850^{\circ} \mathrm{C}$. Para minimizar o efeito da porosidade nos espectros de impedância foram selecionadas amostras sinterizadas a $1000{ }^{\circ} \mathrm{C}$, todas com densidades relativas superiores a $92 \%$. Os espectros de impedância de cerâmicas policristalinas geralmente descrevem uma sequência de semicírculos que da região de altas frequências para a de baixas frequências correspondem a efeitos capacitivos e resistivos associados ao grão, bloqueio de portadores de carga nas interfaces entre os grãos (contornos de grão) e processos eletroquímicos ocorrendo na interface cerâmica/eletrodo. Este comportamento elétrico típico pode ser aplicado às soluções sólidas à base de céria deste estudo. A Fig. 3 apresenta o espectro de impedância obtido a $400{ }^{\circ} \mathrm{C}$ para a amostra de composição $\mathrm{Ce}_{0,99} \mathrm{Cu}_{0,01} \mathrm{O}_{2-\delta}$. Como pode ser visto na Fig. 3a, a amostra é altamente resistiva, sendo a quase totalidade da sua resistividade associada ao efeito bloqueador dos contornos de grão (elevada resistividade de contorno de grão). De fato, a resistividade do grão, relacionada ao semicírculo de alta frequência, é muito pequena comparada com a do contorno de grão (Fig. 3b). Um modelo de circuito elétrico é utilizado para representar os processos ou fenômenos físicos que ocorrem na amostra, tais como contribuições para condutividade relacionadas com o grão e contorno de grão, no caso de eletrólitos sólidos policristalinos. A partir do ajuste do modelo de circuito elétrico é possível analisar e interpretar as medidas obtidas por espectroscopia de impedância e extrair informações como condutividade elétrica e energia de ativação para os processos de condução. $\mathrm{O}$ modelo de circuito elétrico representado por uma associação em série de elementos de circuito RC (resistor/capacitor) foi usado para ajustar os espectros de impedância obtidos. Como poder ser observado na Fig. 4, o espectro da impedância de contorno de grão obtido a $400{ }^{\circ} \mathrm{C}$ para a amostra de composição $\mathrm{Ce}_{0,99} \mathrm{Cu}_{0,01} \mathrm{O}_{2-\delta}$ foi satisfatoriamente ajustado usando o circuito equivalente ilustrado nesta figura. Neste ajuste, o elemento de fase constante (CPE, do inglês constant phase element) é inserido no lugar de um capacitor (C), por ser uma generalização de uma capacitância, levando em consideração a "não idealidade" dos semicírculos observados experimentalmente (semicírculos abatidos).
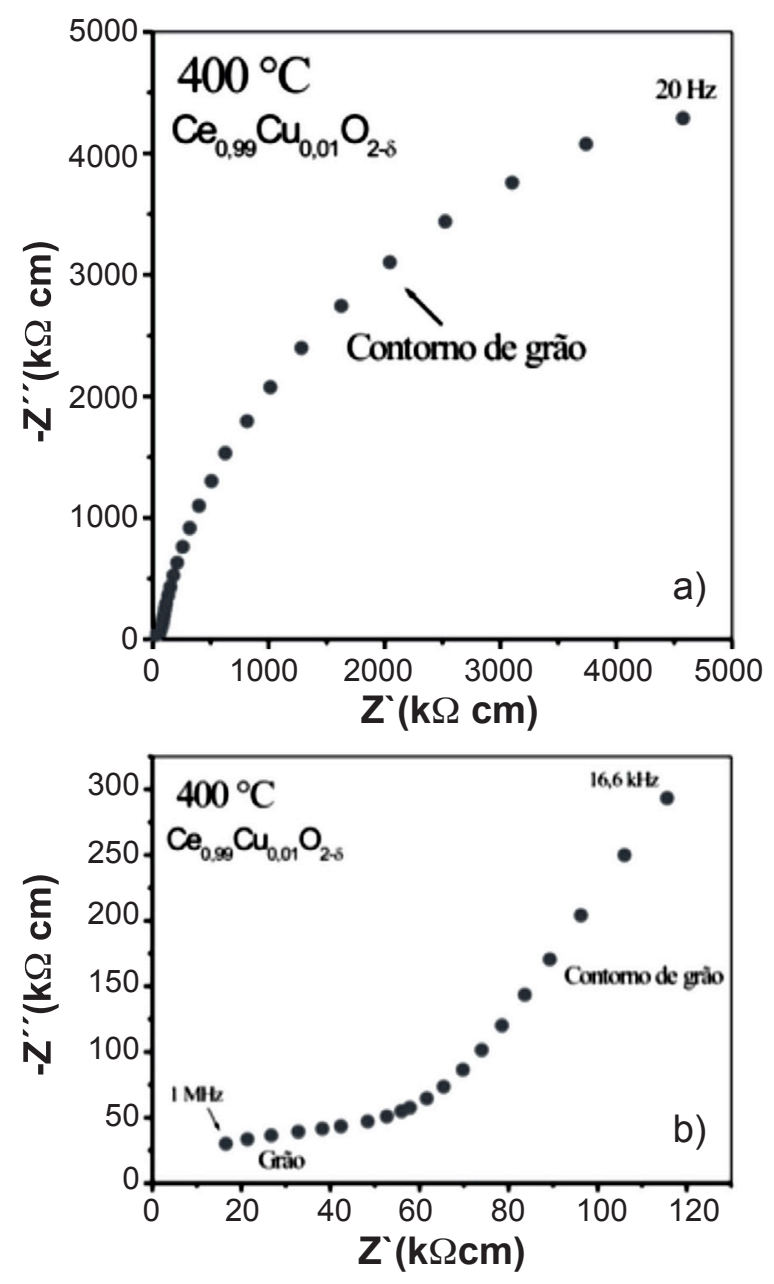

Figura 3: Espectro de impedância a $400{ }^{\circ} \mathrm{C}$ para a amostra $\mathrm{Ce}_{0,99} \mathrm{Cu}_{0,01} \mathrm{O}_{2-\delta}$ sinterizada a $1000{ }^{\circ} \mathrm{C}$ por $5 \mathrm{~h}$ : (a) em toda a faixa de frequência ( $20 \mathrm{~Hz}-1 \mathrm{MHz}$ ) e (b) na região de altas frequências. [Figure 3: Impedance spectrum at $400{ }^{\circ} \mathrm{C}$ for sample $\mathrm{Ce}{ }_{0.99} \mathrm{Cu}_{0.01} \mathrm{O}_{2-\delta}$ sintered at $1000{ }^{\circ} \mathrm{C}$ for $5 \mathrm{~h}:(a)$ between $20 \mathrm{~Hz}$ and $1 \mathrm{MHz}$, and (b) in the high frequency region.] 


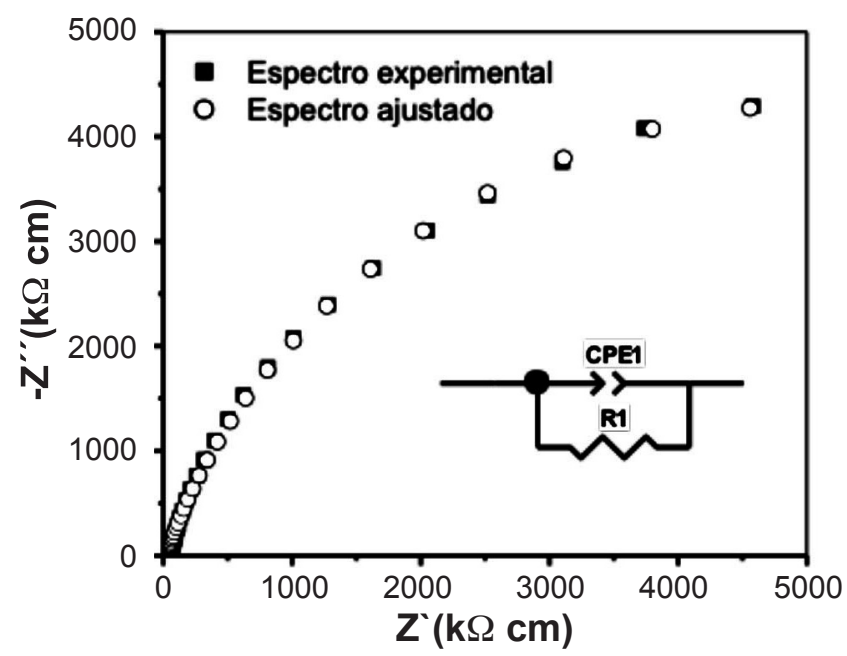

Figura 4: Espectros experimental e ajustado para a impedância de contorno de grão obtida a $400{ }^{\circ} \mathrm{C}$ para a amostra de composição $\mathrm{Ce}_{0,99} \mathrm{Cu}_{0,01} \mathrm{O}_{2-\delta}$.

[Figure 4: Experimental and fitted spectra for the grain boundary impedance obtained at $400{ }^{\circ} \mathrm{C}$ for the sample of composition $\left.\mathrm{Ce}_{0.99} \mathrm{Cu}_{0.01} \mathrm{O}_{2-\delta}.\right]$

A Fig. 5 mostra os espectros de impedância das amostras dopadas com 20 e $30 \%$ em mol de gadolínia, também obtidos a $400{ }^{\circ} \mathrm{C}$. Nestes espectros, corrigidos para as dimensões das amostras, observa-se que as curvas apresentaram semicírculos distintos e que não alcançaram a origem dos eixos ( $Z^{\prime}$, -Z"). As amostras não apresentaram $\left(\mathrm{Ce}_{0,79} \mathrm{Gd}_{0,20} \mathrm{Cu}_{0,01} \mathrm{O}_{2-\delta}\right)$ ou apresentaram apenas parte $\left(\mathrm{Ce}_{0,69} \mathrm{Gd}_{0,30} \mathrm{Cu}_{0,01} \mathrm{O}_{2-\delta}\right)$ dos semicírculos de alta frequência relativos à impedância do grão (I), que neste caso podem ser consideradas simples resistores, cujos valores de resistividade correspondem às distâncias do baixo intercepto dos semicírculos de contorno de grão (II) à origem dos eixos, medidos sobre o eixo da impedância real (Z'). Os semicírculos nas regiões de baixas frequências foram relacionados aos processos que ocorreram na interface entre as cerâmicas e os eletrodos (III), que neste estudo foram filmes porosos de platina. Para maiores temperaturas de medida, espera-se que o semicírculo de alta frequência desapareça completamente, quando os grãos passam a ser somente resistivos. Em temperaturas mais

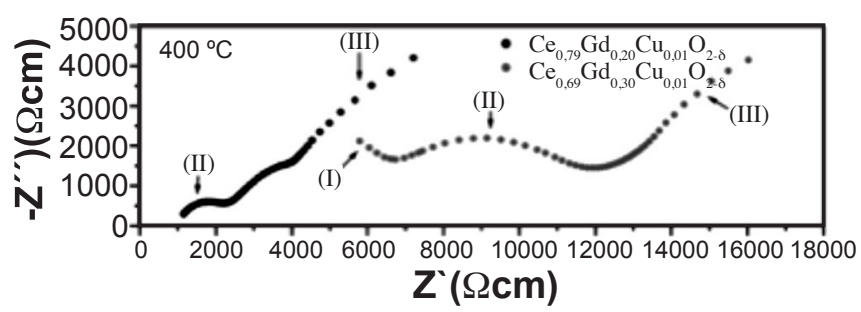

Figura 5: Espectros de impedância a $400{ }^{\circ} \mathrm{C}$ para as amostras dopadas com 20 e $30 \%$ em mol de gadolínia e sinterizadas a 1000 ${ }^{\circ} \mathrm{C}$ por $5 \mathrm{~h}$. As indicações são para os semicírculos do grão (I), contorno de grão (II) e eletrodo (III).

[Figure 5: Impedance spectra at $400{ }^{\circ} \mathrm{C}$ for samples doped with 20 and 30 mol\% of gadolinia and sintered at $1000{ }^{\circ} \mathrm{C}$ for $5 \mathrm{~h}$. Indications are for semicircles of grain (I), grain boundary (II) and electrode (III).] elevadas, normalmente entre 600 e $800{ }^{\circ} \mathrm{C}$, os contornos de grão também passaram a ser somente resistivos (desaparecimento do segundo semicírculo), restando apenas o semicírculo correspondente aos eletrodos, que não foram abordados neste trabalho.

A Fig. 6 apresenta espectros de impedância obtidos a $750{ }^{\circ} \mathrm{C}$, exemplos típicos do comportamento elétrico em altas temperaturas. A presença apenas do semicírculo do eletrodo impossibilita a determinação das resistividades de grão e contorno de grão, sendo possível obter informações apenas sobre a resistividade total $\left(\varrho_{\text {Total }}\right)$, obtida a partir do intercepto com o eixo real na região de alta frequência. Nestas condições, as resistividades totais para as amostras $\mathrm{Ce}_{0,79} \mathrm{Gd}_{0,20} \mathrm{Cu}_{0,01} \mathrm{O}_{2-\delta}$ e $\mathrm{Ce}_{0,69} \mathrm{Gd}_{0,30} \mathrm{Cu}_{0,01} \mathrm{O}_{2-\delta}$, obtidas a $750{ }^{\circ} \mathrm{C}$ (a partir da Fig. 6), apresentaram valores entre 30 e $50 \mathrm{ohm}$. $\mathrm{cm}$, havendo um decréscimo de condutividade total com o aumento do teor de gadolínia de 20 para $30 \%$ em mol.

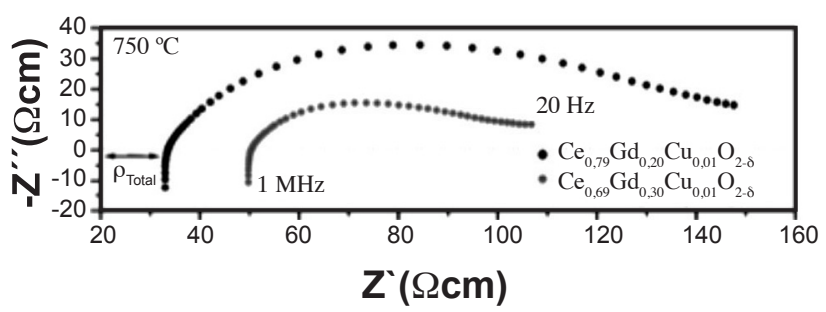

Figura 6: Espectros de impedância obtidos a $750{ }^{\circ} \mathrm{C}$ para as amostras $\mathrm{Ce}_{0,79} \mathrm{Gd}_{0,20} \mathrm{Cu}_{0,01} \mathrm{O}_{2-\delta} \mathrm{e} \mathrm{Ce}_{0,69} \mathrm{Gd}_{0,30} \mathrm{Cu}_{0,01} \mathrm{O}_{2-\delta}$.

[Figure 6: Impedance spectra at $750{ }^{\circ} \mathrm{C}$ for samples $\mathrm{Ce}_{0.79} \mathrm{Gd} d_{0.20} \mathrm{Cu} u_{0.01} \mathrm{O}_{2-\delta}$ and $\mathrm{Ce} e_{0.69} G d_{0.30} \mathrm{Cu}_{0.01} \mathrm{O}_{2-\boldsymbol{\delta}}$. $]$

A Fig. 7 mostra os gráficos de Arrhenius das condutividades totais para as três composições em estudo: $\mathrm{Ce}_{0,99-\mathrm{x}} \mathrm{Gd}_{\mathrm{x}} \mathrm{Cu}_{0,01} \mathrm{O}_{2-\delta} \quad(\mathrm{x}=0,0,2$ e 0,3$)$. A dependência da condutividade com a temperatura, para as amostras codopadas, não pôde ser ajustada para uma única linha reta. As curvas exibiram diferentes inclinações nas regiões de baixa e alta temperatura, com o ponto de transição em

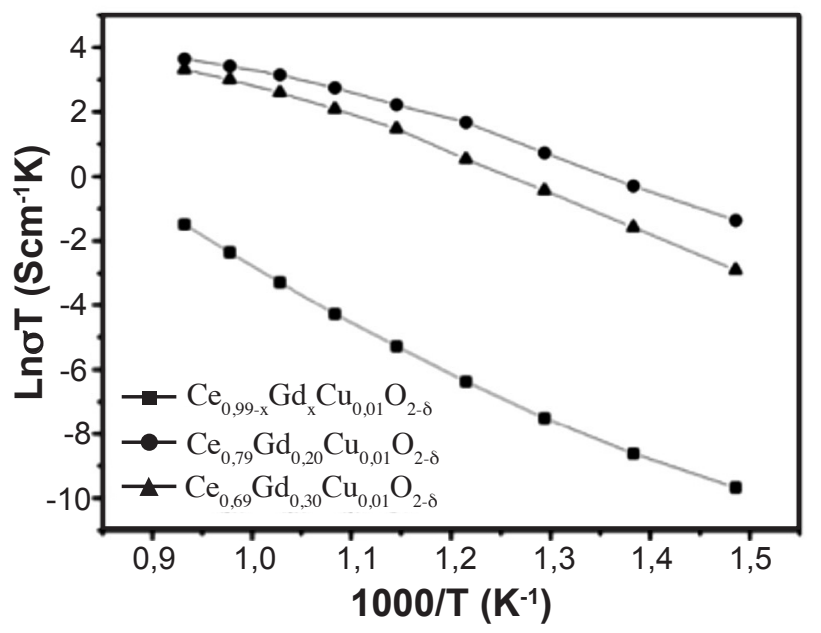

Figura 7: Gráfico de Arrhenius das condutividades totais para as composições $\mathrm{Ce}_{0,99-\mathrm{x}} \mathrm{Gd}_{\mathrm{x}} \mathrm{Cu}_{0,01} \mathrm{O}_{2-\delta}(\mathrm{x}=0,0,2$ e 0,3).

[Figure 7: Arrhenius plot of total conductivity for compositions $C e_{0.99-x} G d_{x} C u_{0.01} O_{2-\delta}(x=0,0.2$ and 0.3).] 
Tabela IV - Condutividade total (a 500 e $700{ }^{\circ} \mathrm{C}$ ) para as composições $\mathrm{Ce}_{0,99-\mathrm{x}} \mathrm{Gd}_{\mathrm{x}} \mathrm{Cu}_{0,01} \mathrm{O}_{2-\delta}(\mathrm{x}=0,0,2$ e 0,3). Dados da literatura são tabulados para comparação.

[Table IV - Total conductivity (at 500 and $700{ }^{\circ} \mathrm{C}$ ) for compositions $\mathrm{Ce}_{0.99-x} \mathrm{Gd} \mathrm{Cu}_{0.01} \mathrm{O}_{2-\delta}(x=0,0.2$ and 0.3). Literature data are tabulated for comparison.]

\begin{tabular}{cccc}
\hline Composição & $\begin{array}{c}\sigma_{500} \\
(\mathrm{~S} / \mathrm{cm})\end{array}$ & $\begin{array}{c}\sigma_{700} \\
(\mathrm{~S} / \mathrm{cm})\end{array}$ & Referência \\
\hline $\mathrm{Ce}_{0,99} \mathrm{Cu}_{0,01} \mathrm{O}_{2-\delta}$ & $7,11 \times 10^{-7}$ & $3,83 \times 10^{-5}$ & Este trabalho \\
$\mathrm{Ce}_{0,79} \mathrm{Gd}_{0,20} \mathrm{Cu}_{0,01} \mathrm{O}_{2-\delta}$ & $2,69 \times 10^{-3}$ & $23,8 \times 10^{-3}$ & Este trabalho \\
$\mathrm{Ce}_{0,69} \mathrm{Gd}_{0,30} \mathrm{Cu}_{0,01} \mathrm{O}_{2-\delta}$ & $0,8 \times 10^{-3}$ & $13 \times 10^{-3}$ & Este trabalho \\
$\mathrm{CeO}_{2-\delta}$ & - & $1,9 \times 10^{-5}$ & {$[28]$} \\
$\mathrm{Ce}_{0,80} \mathrm{Sm}_{0,20} \mathrm{O}_{2-\delta}$ & - & $18 \times 10^{-3}$ & {$[29]$} \\
$\mathrm{Ce}_{0,80} \mathrm{Sm}_{0,20} \mathrm{O}_{2-\delta}$ & $0,53 \times 10^{-3}$ & - & {$[30]$} \\
$\mathrm{Ce}_{0,79} \mathrm{Gd}_{0,20} \mathrm{Zn}_{0,01} \mathrm{O}_{2-\delta}$ & - & $28 \times 10^{-3}$ & {$[31]$} \\
$\mathrm{Ce}_{0,79} \mathrm{Gd}_{0,20} \mathrm{Cu}_{0,01} \mathrm{O}_{2-\delta}$ & - & $>26 \times 10^{-3}$ & {$[32]$} \\
\hline
\end{tabular}

aproximadamente $650{ }^{\circ} \mathrm{C}$. Isto implica que o mecanismo para a condução elétrica mudou a esta temperatura. Em temperaturas acima de $650{ }^{\circ} \mathrm{C}$ a condução elétrica foi controlada pela quantidade de defeitos portadores de carga (vacâncias de oxigênio), que dependeu da concentração de cátions dopantes. Abaixo de $650{ }^{\circ} \mathrm{C}$ a quantidade de portadores de carga foi determinada pelo equilíbrio termodinâmico entre as vacâncias livres e os pares associados (íon dopante-vacância). Assim, a inclinação da linha tornou-se mais acentuada, devido à maior energia de ativação para a condução elétrica abaixo de $650{ }^{\circ} \mathrm{C}$.

De acordo com a Fig. 7, a solução sólida de Ce e $\mathrm{Cu}$ apresentou menores valores de condutividade total, em todo o intervalo de temperatura $\left(400-800^{\circ} \mathrm{C}\right)$, comparativamente às amostras codopadas com gadolínia. Por outro lado, considerável aumento de condutividade foi observado com a adição de 20 a $30 \%$ em mol de $\mathrm{Gd}_{2} \mathrm{O}_{3}$. A literatura relata que a céria pura torna-se bom condutor quando uma fração dos íons de $\mathrm{Ce}^{4+}$ é substituída por íons de valência menor, tais como $\mathrm{Gd}^{3+}$ e $\mathrm{Sm}^{3+}$, atingindo condutividades de 0,082 a $0,1 \mathrm{~S} / \mathrm{cm}$ a $800{ }^{\circ} \mathrm{C}[7,24]$. A melhoria nas propriedades elétricas foi associada à geração de vacâncias de oxigênio, criadas para manter a neutralidade elétrica na rede da céria [25]. Embora as amostras codopadas com $\mathrm{Gd}^{3+}$ tenham apresentado valores de condutividade similares (um pouco superior para a composição $\mathrm{Ce}_{0,79} \mathrm{Gd}_{0,20} \mathrm{Cu}_{0,01} \mathrm{O}_{2-\delta}$ ), especialmente a altas temperaturas, para temperaturas abaixo de $600{ }^{\circ} \mathrm{C}$ houve notável aumento na diferença de condutividade. Os mais altos valores de condutividade elétrica total em cerâmicas de céria dopada com gadolínia ainda é uma matéria de debate. Os valores máximos, reportados para céria contendo entre 15 e $20 \%$ em mol de $\mathrm{Gd}$, tem se mostrado fortemente dependentes das rotas de obtenção de pós e das condições de sinterização [26, 27]. Neste estudo, as grandes contribuições do grão e do contorno de grão para a resistividade da composição
$\mathrm{Ce}_{0,69} \mathrm{Gd}_{0,30} \mathrm{Cu}_{0,01} \mathrm{O}_{2-\delta}$, como visto anteriormente na Fig. 5, levaram a crer que a amostra com $20 \%$ em mol de gadolínia é a melhor opção (apesar da densidade relativa levemente superior para a composição com $30 \%$ de Gd) para aplicação como material de eletrólito em células a combustível de óxido sólido.

A Tabela IV apresenta valores de condutividade total (a 500 e $700{ }^{\circ} \mathrm{C}$ ) e energias de ativação obtidas a partir do gráfico de Arrhenius das condutividades. Uma análise comparativa destes resultados foi feita com dados da literatura. A condutividade da amostra $\mathrm{Ce}_{0,99} \mathrm{Cu}_{0,01} \mathrm{O}_{2-\delta}$ primeiro aumentou e depois diminuiu com a adição de íons de $\mathrm{Gd}^{3+}$, atingindo um máximo de $23,8 \mathrm{mS} / \mathrm{cm}$ a $700{ }^{\circ} \mathrm{C}$ para $20 \%$ em mol de $\mathrm{Gd}_{2} \mathrm{O}_{3}$. A hipótese de que a adição de $1 \%$ em mol de $\mathrm{CuO}$ ao $\mathrm{CeO}_{2}$ não implicaria em melhor desempenho elétrico foi confirmada por comparação com o trabalho [28], no qual foi obtido $1,9 \times 10^{-5} \mathrm{~S} / \mathrm{cm}$ a $700{ }^{\circ} \mathrm{C}$ para a $\mathrm{CeO}_{2-\delta}$. As condutividades da amostra codopada com $20 \%$ em mol de gadolínia ( sinterizada a $1000{ }^{\circ} \mathrm{C}$ ) foram levemente superiores às de cerâmicas de composição $\mathrm{Ce}_{0,80} \mathrm{Sm}_{0,20} \mathrm{O}_{2-\delta}$ sinterizadas a 1275 e $1500{ }^{\circ} \mathrm{C}[29,30]$, comprovando a eficácia do pequeno teor de $\mathrm{CuO}$ como aditivo de sinterização, sem comprometer a condutividade elétrica. Valores similares de condutividade são reportados para céria codopada com outros aditivos [31, 32]. Os resultados obtidos neste trabalho motivaram a estudar processos de cossinterização de bicamadas anodo/eletrólito unindo os materiais obtidos neste trabalho a cermets $\mathrm{Cu}$ SDC preparados por uma metodologia de síntese em uma etapa [33].

\section{CONCLUSÕES}

No presente trabalho foram investigadas as características de materiais particulados e cerâmicas sinterizadas de composições $\mathrm{Ce}_{0,99-\mathrm{x}} \mathrm{Gd}_{\mathrm{x}} \mathrm{Cu}_{0,01} \mathrm{O}_{2-\delta}(\mathrm{x}=0,0,2$ e 0,3 ) sintetizadas pelo método dos precursores poliméricos e sinterizadas entre 950 e $1050^{\circ} \mathrm{C}$. Os resultados de difração de raios $\mathrm{X}$ sugeriram que as sucessivas adições de gadolínia aumentaram o número de vacâncias de oxigênio e, como resultado, conduziram ao aumento do parâmetro de rede. O refinamento Rietveld dos dados de difração indicou a obtenção de partículas nanocristalinas $\left(D_{D R X}<15 \mathrm{~nm}\right)$ de soluções sólidas à base de céria com estrutura cúbica do tipo fluorita após calcinação a $500{ }^{\circ} \mathrm{C}$ por $1 \mathrm{~h}$. A associação de duas metodologias de processamento (nanopartículas + aditivos de sinterização) permitiu consolidar cerâmicas com densidades relativas superiores a $92 \%$ em temperaturas tão baixas quanto $1000{ }^{\circ} \mathrm{C}$, implicando em uma diminuição de pelo menos $400{ }^{\circ} \mathrm{C}$ em comparação a cerâmicas da literatura livres de aditivos de sinterização. Com base nos resultados de espectroscopia de impedância observou-se que a condutividade elétrica foi função do teor de gadolínia, sendo otimizada para $20 \%$ em mol deste dopante.

\section{AGRADECIMENTOS}

Os autores agradecem à CAPES pela bolsa de mestrado de 
Thamyscira H. S. Silva e ao CNPq (processo 447797/2014-0).

\section{REFERÊNCIAS}

[1] A. Choudhury, H. Chandra, A. Arora, Renew. Sust. Energ. Rev. 20 (2013) 430.

[2] A. Atkinson, S. Barnett, R.J. Gorte, J.T.S. Irvine, A.J. McEvoy, M. Mogensen, S.C. Singhal, J. Vohs, Nat. Mater. 3 (2004) 17.

[3] N.Q. Minh, J. Am. Ceram. Soc. 76, 3 (1993) 563.

[4] H. Liu, X. Zhu, M. Cheng, Y. Cong, W. Yang, Int. J. Hydrog. Energ. 38 (2013) 1052.

[5] H. Wendt, M. Götz, M. Linardi, Quím. Nova 23 (2000) 538.

[6] S.C. Singhal, K. Kendall, High-temperature solid oxide fuel cells: fundamentals, design and applications, Elsevier Science, Amsterdam (2004).

[7] B.C.H. Steele, Solid State Ion. 129 (2000) 95.

[8] T.C. Porfirio, "Influência do cálcio e do lítio na sinterização e na condutividade elétrica do óxido de cério contendo gadolínio", Diss. Mestr., IPEN, S. Paulo (2011).

[9] H. Hayashi, H. Inaba, M. Matsuyama, N.G. Lan, M. Dokiya, H. Tagawa, Solid State Ion. 122 (1999) 1.

[10] H. Hayashi, R. Sagawa, H. Inaba, K. Kawamura, Solid State Ion. 131 (2000) 281.

[11] D.Z. Florio, F.C. Fonseca, E.N.S. Muccillo, R. Muccillo, Cerâmica 50, 316 (2004) 275.

[12] G.B. Crochemore, D.P.F. Souza, Rev. Matéria 14, 3 (2009) 1076.

[13] Y. Dong, S. Hampshire, J. Zhou, G. Meng, Int. J. Hydrog. Energ. 36 (2011) 5454.

[14] Z. Khakpour, A.A. Youzbashi, A. Maghsoudipour, K. Ahmadi, Powder Technol. 214 (2011) 117.

[15] V. Gil, J. Tartaj, C. Moure, P. Duran, Ceram. Int. 33 (2007) 471.

[16] L.A. Villas-Bôas, D.P.F. de Souza, Cerâmica 60, 354 (2014) 285.

[17] M.C.F. Dias, E.N.S. Muccillo, Cerâmica 59, 352 (2013)
522.

[18] L. Kleinlongel, L.J. Gauckler, Adv. Mater. 13 (2001) 1081 .

[19] H. Vu, D. Nguyen, J.G. Fisher, W.H. Moon, S. Bae, H.G. Park, B.G. Parkc, J. Asian Ceram. Soc. 1 (2013) 170.

[20] C.G.M. Lima, T.H. Santos, J.P.F. Grilo, R.P.S. Dutra, R.M. Nascimento, S. Rajesh, F.C. Fonseca, D.A. Macedo, Ceram. Int. 41 (2014) 4161.

[21] B. Cela, D.A. Macedo, G.L. Souza, A.E. Martinelli, R.M. Nascimento, C.A. Paskocimas, J. Power Sources 196 (2011) 2539.

[22] R.V. Wandekar, M. Ali, B.N. Wani, S.R. Bharadwa, Mater. Chem. Phys. 99 (2006) 289.

[23] R.O. Fuentes, R.T. Baker, J. Power Sources 186 (2009) 268.

[24] R. Peng, C. Xia, Q. Fu, G. Meng, D. Peng, Mater. Lett. 56 (2002) 1043.

[25] K. Huang, M. Feng, J.B. Goodenough, J. Am. Ceram. Society 81 (1998) 357.

[26] T. Kudo, H. Obayashi, J. Electrochem. Soc. 123 (1976) 415.

[27] S. Zha, C. Xia, G. Meng, J. Power Sources 155 (2003) 44.

[28] W. Huang, P. Shuk, M. Greenblatt, Chem. Mat. 9 (1997) 2240 .

[29] R. Peng, C. Xia, Q. Fu, G. Meng, D. Peng, Mat. Lett. 56 (2002) 1043.

[30] M. Srivastava, K. Kumar, N. Jaiswal, N.K. Singh, D. Kumar, O. Parkash, Ceram. Int. 40 (2014) 10901.

[31] L.A.V. Boas, F.M.L. Figueiredo, D.P.F. Souza, F.M.B. Marques, Solid State Ion. 262 (2014) 522.

[32] Y. Dong, S. Hampshire, B. Lin, Y. Ling, X. Zhang, J. Power Sources 195 (2010) 6510.

[33] H.C.T. Firmino, A.J.M. Araújo, R.P.S. Dutra, R.M. Nascimento, S. Rajesh, D.A. Macedo, Cerâmica, 63, 365 (2017) 52.

(Rec. 08/08/2016, Rev. 21/10/2016, 30/11/2016, Ac. 17/02/2017) 